Journal of Zhejiang University-SCIENCE B (Biomedicine \& Biotechnology) ISSN 1673-1581 (Print); ISSN 1862-1783 (Online)

www.jzus.zju.edu.cn; www.springerlink.com

E-mail: jzus@zju.edu.cn

\title{
Correspondence:
}

\section{Can SARS-CoV-2-infected women breastfeed after viral clearance?*}

\author{
Guan-jing LANG, Hong ZHAO ${ }^{\dagger *}$ \\ Department of Infectious Diseases, State Key Laboratory for the \\ Diagnosis and Treatment of Infectious Diseases, Collaborative Innovation \\ Center for the Diagnosis and Treatment of Infectious Diseases, National \\ Clinical Research Center for Infectious Diseases, the First Affiliated \\ Hospital, School of Medicine, Zhejiang University, Hangzhou 310003, \\ China \\ ${ }^{\dagger}$ E-mail: zjuzhaohong@zju.edu.cn
}

https://doi.org/10.1631/jzus.B2000095

Published online May 8, 2020

The recently emerged novel coronavirus pneumonia, named the coronavirus disease 2019 (COVID19), shares several clinical characteristics with severe acute respiratory syndrome (SARS) and Middle East respiratory syndrome (MERS), and spread rapidly throughout China in December of 2019 (Huang et al., 2020). The pathogen 2019 novel coronavirus (2019$\mathrm{nCoV}$ ) is now named SARS coronavirus 2 (SARSCoV-2) and is highly infectious. As of Apr. 9, 2020, over 80000 confirmed cases had been reported, with an estimated mortality rate of $4.0 \%$ (Chinese Center for Disease Control and Prevention, 2020). Person-toperson transmission and familial clustering have been reported (Chan et al., 2020; Nishiura et al., 2020; Phan et al., 2020). However, there is no evidence of fetal intrauterine infection in pregnant women who have been infected with SARS-CoV-2 in their third trimester (Chen et al., 2020). It is unclear whether breastfeeding transmits the virus from previously infected and recovered mothers to their babies. Here we report the clinical course of a pregnant woman with COVID-19. In order to determine whether SARS-CoV-2 can be transmitted to newborns through breastfeeding, we measured viral RNA in the patient's

\footnotetext{
Corresponding author

* Project supported by the Chinese National Science and Technology Major Project (No. 2017ZX10204401001002)

(D) ORCID: Guan-jing LANG, https://orcid.org/0000-0003-0071-2924

(C) Zhejiang University and Springer-Verlag GmbH Germany, part of Springer Nature 2020
}

breastmilk samples at different time points after delivery.

The patient was a 30 -year-old female (gravida 2, para 1) at 35 weeks and $2 \mathrm{~d}$ of gestation with no underlying medical conditions (Huang et al., 2020). Her husband had a fever on Feb. 1, 2020, and was subsequently diagnosed with COVID-19. On the same day, the patient went to a local hospital complaining of a dry cough, but had no fever, chest tightness, or shortness of breath. However, her sputum tested positive by real-time polymerase chain reaction (RT-PCR) for SARS-CoV-2. She was then transferred from the local hospital to our center, the First Affiliated Hospital of Zhejiang University, Hangzhou, China, on Feb. 6, 2020.

After admission, laboratory testing revealed a slightly elevated C-reactive protein level $(11.76 \mathrm{mg} / \mathrm{L})$ and a normal leukocyte count $\left(8.7 \times 10^{9}\right.$ cells $\left./ \mathrm{L}\right)$, with $81.4 \%$ segmented neutrophils, $12.9 \%$ lymphocytes, and $0.5 \%$ monocytes. Tests for influenza virus type A/B, parainfluenza type I/II/III, respiratory syncytial virus, and adenovirus were all negative. The chest computed tomography images revealed diffuse bilateral lung infiltrates. The patient received the antiviral lopinavir-ritonavir. On the third day of hospitalization, she underwent an emergency delivery by cesarean section due to a decrease in the fetal heart rate to 110 beats/min. At this time, RT-PCR testing of her sputum for SARS-CoV-2 was still positive. She delivered a baby boy with Apgar scores of 9 and 10 at 1 and 5 min post-delivery, respectively. Caution was taken during delivery to avoid contact, droplet, and airborne transmissions of the virus. At delivery, RTPCR tests of maternal serum, urine, stool, cord blood, amniotic fluid, and placenta were negative for SARSCoV-2. An oropharyngeal swab from the newborn baby was obtained immediately after birth, and the result revealed that the infant was negative for SARSCoV-2. The baby was then sent to a negative-pressure isolation room in the Children's Hospital of Zhejiang 
University (Hangzhou, China), and the subsequent tests of oropharyngeal swabs, blood, stool, and urine remained negative for SARS-CoV-2. Beginning on the fourth day of hospitalization, repeated RT-PCR analyses of the mother's sputum and breastmilk were consistently negative for SARS-CoV-2. On Feb. 19, 2020, based on the current expert consensus (Xu et al., 2020), the patient was discharged. A two-week quarantine was then required, and follow-up was needed.

Based on previous reports, life-threatening respiratory illnesses like SARS-CoV and MERS-CoV can be associated with critical maternal illness, spontaneous abortion, stillbirth, and maternal death (Payne et al., 2014; Assiri et al., 2016; Maxwell et al., 2017). According to current reports, none of the pregnant women with SARS-CoV-2 infections have developed severe adverse outcomes. Due to the highly similar sequence and identical angiotensin-converting enzyme 2 (ACE2) entry site of SARS-CoV (Zhou et al., 2020), we should be attentive to the disease course and prognosis of COVID-19 in pregnant patients.

Despite the vertical transmission reported in H1N1 and respiratory syncytial virus (Wong et al., 2004; Takahashi et al., 2011), there are still no reports of maternal-fetal transmission of SARS-CoV-2 or other coronaviruses SARS-CoV and MERS-CoV.

Consistent with the virological testing in other cases of SARS-CoV, Robertson et al. (2004) reported a pregnant patient infected with SARS-CoV at 19 weeks' gestation. She recovered after $15 \mathrm{~d}$ in the hospital, and later delivered a healthy baby in the 38th week of pregnancy. No viral RNA was detected in her breastmilk by RT-PCR, although antibodies to SARS$\mathrm{CoV}$ were positive. Stockman et al. (2004) also reported a case of a pregnant woman with SARS. This woman contracted SARS-CoV in her first trimester. After recovering from SARS, she underwent a cesarean section at 36 weeks' gestation, and her infant was also not infected. Three days after delivery, since SARS-CoV was not detected in the specimens collected at delivery, she breastfed her baby (Schneider et al., 2004). Chen et al. (2020) studied nine pregnant women diagnosed with COVID-19 in their third trimester. All patients underwent a cesarean section, and all nine newborns tested negative for the virus. Breastmilk samples were collected from six postpartum patients after their first lactation, and the samples tested negative for SARS-CoV-2.
Our case also found that the infant was healthy based on multiple repeated tests, and the breastmilk samples showed no evidence of SARS-CoV-2 infection. Moreover, the patient was reexamined one week after discharge, and no viral RNA was detected in her sputum or breastmilk.

Breastfeeding provides benefits to both babies and mothers and it is well supported by public policy. Human milk provides optimal nutrients and helps bolster babies' immune systems to fight and prevent infections. Additionally, mothers who breastfeed have a decreased risk of diabetes mellitus, hypertension, and breast cancer (Schwarz and Nothnagle, 2015). However, for many pregnant women who are infected with severe acute and highly pathogenic infectious diseases such as SARS, MERS, and COVID-19, the safety of breastfeeding is still a concern.

The existing data indicates that SARS-CoV-2 cannot be transmitted through breastmilk. In order to minimize neonatal transmission risk, the mother should be isolated from the neonate until she has recovered from COVID-19 and is considered no longer infectious, which requires a two-week quarantine after hospital discharge. However, too few cases have been studied to clearly define the risks and provide guidance for pregnant women infected with SARS-CoV-2. Additionally, the pregnant women reported were in their third trimester at the onset of COVID-19 infection (Da, 2020), so we were unable to ascertain the possibility of intrauterine vertical transmission during the first or second trimester. More data from randomized and controlled multicenter studies are needed to rigorously assess the risks of breastfeeding.

In conclusion, our results suggest that breastfeeding can be practiced after an isolation period is completed and repeat testing is normal. In the meantime, breast pumping is suggested so that breastfeeding may begin once the isolation period ends.

\section{Contributors}

Guan-jing LANG and Hong ZHAO participated in the study design. Guan-jing LANG collected the data and wrote the manuscript. Hong ZHAO edited the manuscript. Both authors have read and approved the final manuscript and, therefore, have full access to all the data in the study and take responsibility for the integrity and security of the data.

\section{Acknowledgments}

We appreciate all clinical providers, nurses, and scientific researchers for their efforts in fighting COVID-19. 


\section{Compliance with ethics guidelines}

Guan-jing LANG and Hong ZHAO declare that they have no conflict of interest.

All procedures followed were in accordance with the ethical standards of the responsible committee on human experimentation (institutional and national) and with the Helsinki Declaration of 1975, as revised in 2008 (5). Informed consent was obtained from the patient for being included in the study.

\section{References}

Assiri A, Abedi GR, al Masri M, et al., 2016. Middle East respiratory syndrome coronavirus infection during pregnancy: a report of 5 cases from Saudi Arabia. Clin Infect Dis, 63(7):951-953. https://doi.org/10.1093/cid/ciw412

Chan JFW, Yuan SF, Kok KH, et al., 2020. A familial cluster of pneumonia associated with the 2019 novel coronavirus indicating person-to-person transmission: a study of a family cluster. Lancet, 395(10223):514-523. https://doi.org/10.1016/S0140-6736(20)30154-9

Chen HJ, Guo JJ, Wang C, et al., 2020. Clinical characteristics and intrauterine vertical transmission potential of COVID-19 infection in nine pregnant women: a retrospective review of medical records. Lancet, 395(10226):809-815. https://doi.org/10.1016/S0140-6736(20)30360-3

Chinese Center for Disease Control and Prevention, 2020. Update on the new coronavirus pneumonia outbreak as of 24:00 on 8 April. http://www.chinacdc.cn/jkzt/crb/zl/szkb 11803/jszl_11809/202004/t20200409_215861.html] [Accessed on Apr. 9, 2020] (in Chinese).

Da S, 2020. An analysis of 38 pregnant women with COVID-19, their newborn infants, and maternal-fetal transmission of SARS-CoV-2: maternal coronavirus infections and pregnancy outcomes. Arch Pathol Lab Med, in press. https://doi.org/10.5858/arpa.2020-0901-SA

Huang CL, Wang YM, Li XW, et al., 2020. Clinical features of patients infected with 2019 novel coronavirus in Wuhan, China. Lancet, 395(10223):497-506. https://doi.org/10.1016/S0140-6736(20)30183-5

Huang JW, Zhou XY, Lu SJ, et al., 2020. Dialectical behavior therapy-based psychological intervention for woman in late pregnancy and early postpartum suffering from COVID-19: a case report. J Zhejiang Univ-Sci B (Biomed \& Biotechnol), 21(5):394-399. https://doi.org/10.1631/jzus.B2010012

Maxwell C, McGeer A, Tai KFY, et al., 2017. No. 225management guidelines for obstetric patients and neonates born to mothers with suspected or probable severe acute respiratory syndrome (SARS). J Obstet Gynaecol Can, 39(8):e130-e137. https://doi.org/10.1016/j.jogc.2017.04.024

Nishiura H, Jung SM, Linton NM, et al., 2020. The extent of transmission of novel coronavirus in Wuhan, China, 2020. J Clin Med, 9(2):330. https://doi.org/10.3390/jcm9020330

Payne DC, Iblan I, Alqasrawi S, et al., 2014. Stillbirth during infection with Middle East respiratory syndrome coronavirus. J Infect Dis, 209(12):1870-1872.

https://doi.org/10.1093/infdis/jiu068

Phan LT, Nguyen TV, Luong QC, et al., 2020. Importation and human-to-human transmission of a novel coronavirus in Vietnam. N Engl J Med, 382(9):872-874. https://doi.org/10.1056/NEJMc2001272

Robertson CA, Lowther SA, Birch T, et al., 2004. SARS and pregnancy: a case report. Emerg Infect Dis, 10(2):345-348. https://doi.org/10.3201/eid1002.030736

Schneider E, Duncan D, Reiken M, et al., 2004. SARS in pregnancy. AWHONN Lifelines, 8(2):122-128. https://doi.org/10.1177/1091592304265557

Schwarz EB, Nothnagle M, 2015. The maternal health benefits of breastfeeding. Am Fam Physician, 91(9):603-604.

Stockman LJ, Lowther SA, Coy K, et al., 2004. SARS during pregnancy, United States. Emerg Infect Dis, 10(9):16891690. https://doi.org/10.3201/eid1009.040244

Takahashi N, Kitajima H, Kusuda S, et al., 2011. Pandemic (H1N1) 2009 in neonates, Japan. Emerg Infect Dis, 17(9): 1763-1765. https://doi.org/10.3201/eid1709.101803

Wong SF, Chow KM, Leung TN, et al., 2004. Pregnancy and perinatal outcomes of women with severe acute respiratory syndrome. Am J Obstet Gynecol, 191(1):292-297. https://doi.org/10.1016/j.ajog.2003.11.019

$\mathrm{Xu} \mathrm{KJ}$, Cai HL, Shen YH, et al., 2020. Management of corona virus disease-19 (COVID-19): the Zhejiang experience. $J$ Zhejiang Univ: Med Sci, in press (in Chinese). https://doi.org/10.3785/j.issn.1008-9292.2020.02.02

Zhou P, Yang XL, Wang XG, et al., 2020. A pneumonia outbreak associated with a new coronavirus of probable bat origin. Nature, 579(7798):270-273.

https://doi.org/10.1038/s41586-020-2012-7

\section{中文概要}

题 目: 新型冠状病毒感染后治愈的女性可以进行母乳喂 养吗?

概 要: 浙江大学医学院附属第一医院感染科收治一名新 型冠状病毒 (SARS-CoV-2) 感染的妊娠晚期孕 妇。在其住院期间, 患者进行了剖腹产术, 新生 儿被证实无 SARS-CoV-2 感染。结合已有的相关 文献报道, SARS-CoV-2 通过母婴垂直传播的依 据不足, 同时乳汁的病毒检测阴性, 因此目前不 能证明 SARS-CoV-2 病毒能通过乳汁传播。通过 研究, 我们建议感染 SARS-CoV-2 的孕妇, 在治 愈出院后, 应经过两周隔离观察, 并且病毒复查 结果为阴性, 才可以进行母乳喂养。

关键词: 新型冠状病毒 (SARS-CoV-2) ; 孕妇; 哺乳; 母婴传播 